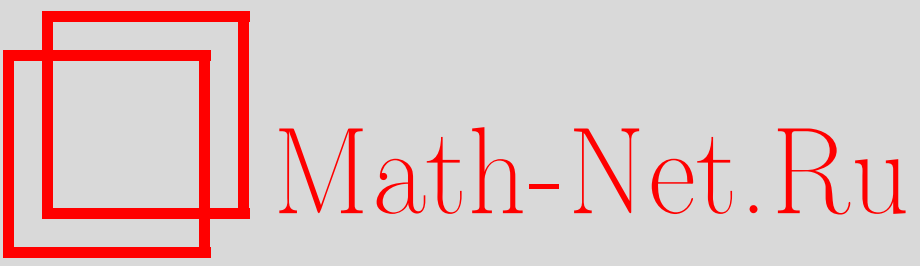

Г. М. Фельдман, Независимые линейные статистики на а-адических соленоидах, Теория вероятн. и ее примен., 2009, том 54, выпуск 3, 515-532

DOI: https://doi.org/10.4213/tvp2807

Использование Общероссийского математического портала Math-Net.Ru подразумевает, что вы прочитали и согласны с пользовательским соглашением

http://www . mathnet.ru/rus/agreement

Параметры загрузки:

IP : 54.92 .164 .108

26 апреля 2023 г., 17:08:18 


\title{
НЕЗАВИСИМЫЕ ЛИНЕЙНЫЕ СТАТИСТИКИ НА а-АДИЧЕСКИХ СОЛЕНОИДАХ
}

\begin{abstract}
Пусть $\xi_{1}$ и $\xi_{2}$ - независимые случайные величины со значениями в а-адическом соленоиде $\Sigma_{\mathbf{a}}$ и с распределениями $\mu_{1}, \mu_{2}$. Пусть $\alpha_{j}, \beta_{j}$ - топологические автоморфизмы группы $\Sigma_{\mathbf{a}}$. В предположении, что линейные статистики $L_{1}=\alpha_{1} \xi_{1}+\alpha_{2} \xi_{2}$ и $L_{2}=\beta_{1} \xi_{1}+\beta_{2} \xi_{2}$ являются независимыми, в статье описываются возможные распределения $\mu_{1}$ и $\mu_{2}$.
\end{abstract}

Ключевые слова и фразы: независимые линейные статистики, а-адический соленоид, топологический автоморфизм.

1. Введение. Приводимая ниже теорема, доказанная независимо В. П. Скитовичем [1] и Ж. Дармуа [2], является одной из наиболее известных характеризационных теорем.

Теорема 1 (Скитович-Дармуа). Пусть $\xi_{j}, j=1, \ldots, n, n \geqslant 2,-$ независимье случайные величины, а $\alpha_{j}, \beta_{j}$ - ненулевые вещественнье иисла. Если линейнье статистики $L_{1}=\alpha_{1} \xi_{1}+\cdots+\alpha_{n} \xi_{n}$ и $L_{2}=\beta_{1} \xi_{1}+$ $\cdots+\beta_{n} \xi_{n}$ независимь, то случайнье величинь $\xi_{j}-$ гауссовские.

Эта теорема была обобщена С. Гурье и И. Олкином на случай, когда $\xi_{j}$ - независимые случайные векторы в пространстве $\mathbf{R}^{m}$, а $\alpha_{j}$, $\beta_{j}$ - несингулярные матрицы. Они доказали, что из независимости $L_{1}$ и $L_{2}$ следует, что случайные векторы $\xi_{j}$ - гауссовские ([3], см. также $[4$, гл. 3]). Большое число исследований было посвящено обобщению теоремы Скитовича-Дармуа на различные алгебраические структуры, в первую очередь на локально компактные абелевы группы (см., например, [5]-[14]). Исследования, стимулированные теоремой СкитовичаДармуа, продолжаются и в классической ситуации [15].

Пусть $X$ - локально компактная сепарабельная абелева метрическая группа, $\operatorname{Aut}(X)$ - группа топологических автоморфизмов $X$, $\Gamma(X)$ - множество гауссовских распределений на $X, I(X)$ - множество идемпотентных распределений на $X$, т.е. множество сдвигов распределений Хаара $m_{K}$ компактных подгрупп $K$ группы $X$. Пусть

*Физико-технический институт низких температур им. Б. И. Веркина НАН Украины, пр-т Ленина, 47, 61103 Харьков, Украина; e-mail: feldman@ilt.kharkov.ua 
$\xi_{j}, j=1, \ldots, n, n \geqslant 2$, - независимые случайные величины со значениями в $X$ и с распределениями $\mu_{j}$. Рассмотрим линейные статистики $L_{1}=\alpha_{1} \xi_{1}+\cdots+\alpha_{n} \xi_{n}$ и $L_{2}=\beta_{1} \xi_{1}+\cdots+\beta_{n} \xi_{n}, n \geqslant 2$, где $\alpha_{j}$, $\beta_{j} \in \operatorname{Aut}(X)$. В последнее время особое внимание уделялось следующей задаче: для каких групп $X$ из независимости $L_{1}$ и $L_{2}$ следует, что все распределения $\mu_{j}$ принадлежит $\Gamma(X) * I(X)$ ? Отметим, что если $\mu \in \Gamma(X) * I(X)$, т.е. $\mu=\gamma * m_{K}$, где $\gamma \in \Gamma(X)$, то $\mu$ инвариантно относительно компактной подгруппы $K \subset X$ и при естественном гомоморфизме $X \mapsto X / K$ индуцирует на фактор-группе $X / K$ гауссовское распределение. Эта задача была решена в статьях [6]-[8] в классах конечных абелевых групп, компактных абелевых групп и дискретных периодических абелевых групп соответственно.

Целью настоящей статьи является изучение следующего вопроса, непосредственно связанного с сформулированной выше задачей. Как было доказано в [10], если $X$ - произвольная связная компактная абелева группа, то существуют такие независимые случайные величины $\xi_{1}$, $\xi_{2}$ со значениями в группе $X$ и с распределениями $\mu_{1}, \mu_{2}$ и такие автоморфизмы $\alpha_{j}, \beta_{j} \in \operatorname{Aut}(X)$, что линейные статистики $L_{1}=\alpha_{1} \xi_{1}+\alpha_{2} \xi_{2}$ и $L_{2}=\beta_{1} \xi_{1}+\beta_{2} \xi_{2}$ независимы, а $\mu_{1}, \mu_{2} \notin \Gamma(X) * I(X)$. Пусть $\xi_{1}, \xi_{2}-$ независимые случайные величины со значениями в группе $X$ и с распределениями $\mu_{1}, \mu_{2}$. Пусть $\alpha_{j}, \beta_{j} \in \operatorname{Aut}(X)$ и предположим, что линейные статистики $L_{1}=\alpha_{1} \xi_{1}+\alpha_{2} \xi_{2}$ и $L_{2}=\beta_{1} \xi_{1}+\beta_{2} \xi_{2}$ независимы. Возникает следующая естественная задача: описать возможные распределения $\mu_{1}$, $\mu_{2}$. Как отмечено выше, $\mu_{j}$ не всегда являются свертками гауссовских и идемпотентных распределений.

Заметим, что если $X$ - связная компактная абелева группа размерности один, то $X$ топологически изоморфна либо группе вращений окружности $\mathbf{T}$ (одномерному тору), либо а-адическому соленоиду $\Sigma_{\mathbf{a}}$. Если $X=\mathbf{T}$, то, не ограничивая общности, можно считать, что линейные статистики имеют вид $L_{1}=\xi_{1}+\xi_{2}, L_{2}=\xi_{1}-\xi_{2}$, и соответствующие распределения $\mu_{j}$ описаны в работе [16]. В настоящей статье мы описываем распределения $\mu_{j}$ для произвольного а-адического соленоида $X=\Sigma_{\mathbf{a}}$ (теорема 1$)$. Ответ оказывается довольно сложным и зависит как от вида соленоида $\Sigma_{\mathbf{a}}$, так и от автоморфизмов $\alpha_{j}, \beta_{j} \in \operatorname{Aut}\left(\Sigma_{\mathbf{a}}\right)$. Тем самым, сформулированная выше задача оказывается решенной для произвольных связных локально компактных абелевых групп размерности один, поскольку если $X-$ связная локально компактная некомпактная абелева группа размерности один, то $X \approx \mathbf{R}$, и решение задачи вытекает из теоремы Скитовича-Дармуа. Отметим также, что при дополнительном условии: характеристические функции распределений $\mu_{j}$ не обращаются в нуль, в [6] было доказано, что на группе $X=\Sigma_{\mathbf{a}}$ из независимости $L_{1}$ и $L_{2}$ следует, что все $\mu_{j}$ гауссовские. 
2. Определения и обозначения. Прежде чем сформулировать основную теорему, напомним некоторые определения и условимся об обозначениях. Для произвольной локально компактной абелевой группы $X$ пусть $Y=X^{*}$ - ее группа характеров, $(x, y)$ - значение характера $y \in Y$ на элементе $x \in X$. Если $K-$ замкнутая подгруппа в $X$, то обозначим через $A(Y, K)=\{y \in Y:(x, y)=1$ для всех $x \in K\}$ ее аннулятор. Если $\delta: X \mapsto X$ - непрерывный гомоморфизм, то сопряженный гомоморфизм $\widetilde{\delta}: Y \mapsto Y$ определяется формулой $(x, \widetilde{\delta} y)=(\delta x, y)$ для всех $x \in X, y \in Y$. Отметим, что $\delta \in \operatorname{Aut}(X)$ тогда и только тогда, когда $\widetilde{\delta} \in \operatorname{Aut}(Y)$. Если $n-$ целое, $n \neq 0$, то $f_{n}$ обозначает гомоморфизм $f_{n}: X \mapsto X$, определяемый формулой $f_{n}(x)=n x$. Положим $X^{(n)}=f_{n}(X)$. Ясно, что $\widetilde{f}_{n}=f_{n}$. Обозначим $\mathbf{Z}$ группу целых чисел, $\mathbf{Q}$ - рассматриваемую в дискретной топологии группу рациональных чисел, $\mathbf{Z}(n)$ - конечную циклическую группу порядка $n$.

Пусть $\mathbf{a}=\left(a_{0}, a_{1}, \ldots\right)$, где все $a_{j}$ принадлежат $\mathbf{Z}, a_{j}>1$. Вначале мы определим группу целых а-адических чисел $\Delta_{\mathbf{a}}$. Как множество $\Delta_{\mathbf{a}}$ совпадает с декартовым произведением $\prod_{n=0}^{\infty}\left\{0,1, \ldots, a_{n}-1\right\}$. Рассмотрим $\mathbf{x}=\left(x_{0}, x_{1}, x_{2}, \ldots\right), \mathbf{y}=\left(y_{0}, y_{1}, y_{2}, \ldots\right) \in \Delta_{\mathbf{a}}$ и определим сумму $\mathbf{z}=\mathbf{x}+\mathbf{y}$ следующим образом. Пусть $x_{0}+y_{0}=t_{0} a_{0}+z_{0}$, где $z_{0} \in\left\{0,1, \ldots, a_{0}-1\right\}$, $t_{0} \in\{0,1\}$. Предположим, что числа $z_{0}, z_{1}, \ldots, z_{k}$ и $t_{0}, t_{1}, \ldots, t_{k}$ уже определены. Положим тогда $x_{k+1}+y_{k+1}+t_{k}=t_{k+1} a_{k+1}+z_{k+1}$, где $z_{k+1} \in$ $\left\{0,1, \ldots, a_{k+1}-1\right\}, t_{k+1} \in\{0,1\}$. Таким образом по индукции определена последовательность $\mathbf{z}=\left(z_{0}, z_{1}, z_{2}, \ldots\right)$. Множество $\Delta_{\mathbf{a}}$ с определенным выше сложением является абелевой группой, нейтральный элемент которой - последовательность в $\Delta_{\mathbf{a}}$, состоящая из нулей. Рассмотрим $\Delta_{\mathbf{a}}$ в топологии произведения. Полученная группа называется группой целых a-адических чисел. Рассмотрим группу $\mathbf{R} \times \Delta_{\mathbf{a}}$. Пусть $B-$ подгруппа в $\mathbf{R} \times \Delta_{\mathbf{a}}$ вида $B=\{(n, n \mathbf{u})\}_{\mathbf{n}=-\infty}^{\infty}$, где $\mathbf{u}=(\mathbf{1}, \mathbf{0}, \ldots, \mathbf{0}, \ldots)$. Факторгруппа $\Sigma_{\mathbf{a}}=\left(\mathbf{R} \times \Delta_{\mathbf{a}}\right) / B$ называется а-адическим соленоидом. Группа $\Sigma_{\mathbf{a}}$ компактна, связна и имеет размерность один (см. $[18,(10.12),(10.13)$, (24.28)]). Группа характеров группы $\Sigma_{\mathbf{a}}$ топологически изоморфна подгруппе $H_{\mathbf{a}} \subset \mathbf{Q}$ вида

$$
H_{\mathbf{a}}=\left\{\frac{m}{a_{0} a_{1} \cdots a_{n}}: \quad n=0,1, \ldots ; m \in \mathbf{Z}\right\} .
$$

Не ограничивая общности, мы будем считать, что если $X=\Sigma_{\mathbf{a}}$, то $Y=X^{*}=H_{\mathrm{a}}$.

Пусть $M^{1}(X)$ - сверточная полугруппа вероятностных распределений на $X, \widehat{\mu}(y)=\int_{X}(x, y) d \mu(x)$ - характеристическая функция распределения $\mu \in M^{1}(X), \sigma(\mu)$ - носитель $\mu$. Если $K$ - замкнутая подгруппа в $X$ и $\sigma(\mu) \subset K$, то $\widehat{\mu}(y+h)=\widehat{\mu}(y)$ для всех $y \in Y, h \in A(Y, K)$. Если $E-$ замкнутая подгруппа в $Y$ и $\widehat{\mu}(y) \equiv 1$ при $y \in E$, то $\widehat{\mu}(y+h)=\widehat{\mu}(y)$ при всех $y \in Y, h \in E$ и $\sigma(\mu) \subset A(X, E)$. Для $\mu \in M^{1}(X)$ через $\bar{\mu} \in M^{1}(X)$ 
обозначим распределение, определяемое формулой $\bar{\mu}(B)=\mu(-B)$ для любого борелевского подмножества $B \subset X$. Заметим, что $\widehat{\bar{\mu}}(y)=\overline{\widehat{\mu}}(y)$.

Распределение $\gamma \in M^{1}(X)$ называется гауссовским [17], если его характеристическая функция представима в виде

$$
\widehat{\gamma}(y)=(x, y) \exp \{-\varphi(y)\},
$$

где $x \in X$, а $\varphi(y)$ - непрерывная неотрицательная функция на $Y$, удовлетворяющая уравнению

$$
\varphi(u+v)+\varphi(u-v)=2[\varphi(u)+\varphi(v)], \quad u, v \in Y .
$$

Отметим, что характеристическая функция распределения Хаара $m_{K}$, где $K$ - компактная подгруппа в $X$, имеет вид

$$
\widehat{m}_{K}(y)= \begin{cases}1, & y \in A(Y, K) \\ 0, & y \notin A(Y, K)\end{cases}
$$

Пусть $\xi_{1}, \xi_{2}$ - независимые случайные величины со значениями в группе $X=\Sigma_{\mathbf{a}}$ и с распределениями $\mu_{1}, \mu_{2}$. Рассмотрим линейные статистики $L_{1}=\alpha_{1} \xi_{1}+\alpha_{2} \xi_{2}$ и $L_{2}=\beta_{1} \xi_{1}+\beta_{2} \xi_{2}$, где $\alpha_{j}, \beta_{j} \in \operatorname{Aut}(X)$. Предположим, что $L_{1}$ и $L_{2}$ независимы. Вводя новые независимые случайные величины $\xi_{j}^{\prime}=\alpha_{j} \xi_{j}, j=1,2$, можно считать, что $L_{1}=\xi_{1}+\xi_{2}$ и $L_{2}=\delta_{1} \xi_{1}+\delta_{2} \xi_{2}$, где $\delta_{j} \in \operatorname{Aut}(X)$. Заметим, что любой топологический автоморфизм $\delta$ группы $X$ имеет вид

$$
\delta=f_{p} f_{q}^{-1}
$$

при некоторых взаимно простых $p$ и $q$, где $f_{p}, f_{q} \in \operatorname{Aut}(X)$. Поскольку при любом $\delta \in \operatorname{Aut}(X)$ линейные статистики $L_{1}$ и $L_{2}$ независимы тогда и только тогда, когда независимы $L_{1}$ и $\delta L_{2}$, то, не ограничивая общности, можно с самого начала предполагать, что $L_{1}=\xi_{1}+\xi_{2}, L_{2}=p \xi_{1}+q \xi_{2}$, где $p, q \in \mathbf{Z}, p q \neq 0, p q \neq 1$ и $p$ и $q$ взаимно просты. Сформулируем теперь основной результат статьи.

Теорема 2. Пусть $X=\Sigma_{\mathbf{a}}, \xi_{1}, \xi_{2}$ - независимье случайные величины со значениями в $X$ и с распределениями $\mu_{1}, \mu_{2}$. Предположим, что $f_{p}, f_{q} \in \operatorname{Aut}(X), p q \neq 1$ и $p$ u $q$ - взаимно просты. Обозначим $s=p-q$, разложим $|s|$ на простые множители, $|s|=s_{1}^{k_{1}} \cdots s_{l}^{k_{l}}$ и рассмотрим соответствуюшие гомоморфизмь $f_{s_{j}}, j=1, \ldots, l$. Если линейные статистики $L_{1}=\xi_{1}+\xi_{2}$ и $L_{2}=p \xi_{1}+q \xi_{2}$ независимь, то возможные распределения $\mu_{1}$ и $\mu_{2}$ описываются в таблицах 1 и 2 . 
Таблица 1.

\begin{tabular}{|c|c|c|}
\hline \multirow{2}{*}{$p q-$ составное } & \multicolumn{2}{|c|}{$p q=-1$} \\
\cline { 2 - 3 } & $f_{2} \in \operatorname{Aut}(X)$ & $f_{2} \notin \operatorname{Aut}(X)$ \\
\hline существуют $\mu_{1}, \mu_{2} \notin \Gamma(X) * I(X)$ & $\mu_{1}, \mu_{2} \in \Gamma(X) * I(X)$ & $\begin{array}{c}\text { существуют } \\
\mu_{1}, \mu_{2} \notin \Gamma(X) * I(X)\end{array}$ \\
\hline
\end{tabular}

Таблица 2.

\begin{tabular}{|c|c|c|c|}
\hline \multicolumn{4}{|c|}{$p q-$ простое } \\
\hline \multirow{2}{*}{$\begin{array}{c}f_{s_{j}} \notin \operatorname{Aut}(X) \\
\text { хотя бы при одном } \\
\qquad s_{j} \neq 2\end{array}$} & \multicolumn{2}{|c|}{$\begin{array}{c}f_{s_{j}} \in \operatorname{Aut}(X) \text { при всех } \\
s_{j} \neq 2 \text { и } f_{2} \notin \operatorname{Aut}(X)\end{array}$} & \multirow[t]{2}{*}{$f_{s} \in \operatorname{Aut}(X)$} \\
\hline & $s$ делится на 4 & $s$ не делится на 4 & \\
\hline $\begin{array}{c}\text { существуют } \\
\mu_{1}, \mu_{2} \notin \Gamma(X) * I(X)\end{array}$ & $\begin{array}{c}\text { существуют } \\
\mu_{1}, \mu_{2} \notin \Gamma(X) * I(X)\end{array}$ & $\begin{array}{c}\text { по крайней } \\
\text { мере одно из } \\
\mu_{j} \in \Gamma(X) * I(X)\end{array}$ & $\begin{array}{c}\text { по крайней } \\
\text { мере одно из } \\
\mu_{j} \in \Gamma(X) * I(X)\end{array}$ \\
\hline
\end{tabular}

Прежде чем перейти к доказательству теоремы 1 , введем следующие обозначения, которые сохраним на протяжении всей статьи. Пусть $f_{s_{j_{1}}}, \ldots, f_{s_{j_{r}}}$ - все гомоморфизмы, которые являются топологическими автоморфизмами $X$. Обозначим через $H$ подгруппу в $Y$ вида

$$
H=\left\{\frac{m}{s_{j_{1}}^{n_{1}} \cdots s_{j_{r}}^{n_{r}}}: \quad m, n_{j} \in \mathbf{Z}\right\}
$$

(в случае, когда $|s|=1$ либо все $f_{s_{j}} \notin \operatorname{Aut}(X)$, считаем, что $H=\mathbf{Z}$ ). Обозначим $G=H^{*}$.

3. Доказательство теоремы 1. Принимая во внимание, что $\widehat{\mu}_{j}(y)=\mathbf{E}\left[\left(\xi_{j}, y\right)\right]$, аналогично тому, как это делается в классическом случае, легко видеть, что независимость линейных статистик $L_{1}$ и $L_{2}$ равносильна тому, что характеристические функции $\widehat{\mu}_{j}(y)$ удовлетворяют функциональному уравнению

$$
\widehat{\mu}_{1}(u+p v) \widehat{\mu}_{2}(u+q v)=\widehat{\mu}_{1}(u) \widehat{\mu}_{2}(u) \widehat{\mu}_{1}(p v) \widehat{\mu}_{2}(q v), \quad u, v \in Y .
$$

Возможные решения этого уравнения мы и будем изучать.

Случай 1: pq-составное.

Докажем, что в этом случае существуют независимые случайные величины $\xi_{1}, \xi_{2}$ со значениями в $X$ и с распределениями $\mu_{1}, \mu_{2}$ такие, что линейные статистики $L_{1}$ и $L_{2}$ независимы, а $\mu_{1}, \mu_{2} \notin \Gamma(X) * I(X)$. Очевидно, что, не ограничивая общности, можно считать выполненным следующее. Если $y=m / n \in Y, m, n \in \mathbf{Z}, n \neq 0$, то в разложении $n$ на простые множители участвуют лишь те $s_{j}$ из разложения $|s|$, для 
которых $f_{s_{j}} \in \operatorname{Aut}(X)$. Поэтому из $s y \in H$ следует, что $y \in H$. Имеются две возможности.

А. $|p|>1,|q|>1$. Поскольку $p$ и $s$, а также $q$ и $s$ взаимно просты, то $H^{(p)} \neq H$ и $H^{(q)} \neq H$. Пусть $\lambda_{j} \in M^{1}(G)$ и $\sigma\left(\lambda_{1}\right) \subset A\left(G, H^{(p)}\right)$, $\sigma\left(\lambda_{2}\right) \subset A\left(G, H^{(q)}\right)$. Тогда $\widehat{\lambda}_{1}(u+p v)=\widehat{\lambda}_{1}(u), \widehat{\lambda}_{2}(u+q v)=\widehat{\lambda}_{2}(u)$ при $u, v \in H$. Отсюда, очевидно, следует, что характеристические функции $\widehat{\lambda}_{j}(y)$ удовлетворяют уравнению (2) при $u, v \in H$. Положим

$$
g_{j}(y)= \begin{cases}\widehat{\lambda}_{j}(y), & y \in H \\ 0, & y \notin H\end{cases}
$$

Функции $g_{j}(y)$ являются положительно определенными на $Y[19, \S 32]$. По теореме Бохнера существуют такие распределения $\mu_{j} \in M^{1}(X)$, что $\widehat{\mu}_{j}(y)=g_{j}(y), j=1,2$. Проверим, что характеристические функции $\widehat{\mu}_{j}(y)$ удовлетворяют уравнению (2). Очевидно, что если $u, v \in H$, то (2) выполнено. Пусть $u \notin H$. Тогда правая часть в (2) равна нулю. Если при этом левая часть в (2) отлична от нуля, то

$$
u+p v \in H \quad \text { и } \quad u+q v \in H .
$$

Но из (4) тогда следует, что $s v \in H$. Значит, $v \in H$, и поэтому $u \in H$, что противоречит выбору $u$. Следовательно, и левая часть в (2) равна нулю. Пусть $v \notin H$. Если $p v, q v \in H$, то и $s v \in H$. Отсюда следует, что $v \in H$, что противоречит выбору $v$. Значит, либо $p v \notin H$, либо $q v \notin H$. Тогда правая часть в (2) равна нулю. Если при этом левая часть в (2) отлична от нуля, то выполнено (4), что влечет $v \in H$, вопреки выбору $v$. Значит, левая часть в (2) равна нулю.

Итак, уравнение (2) справедливо при любых $u, v \in Y$, а следовательно, линейные статистики $L_{1}$ и $L_{2}$ независимы. Ясно, что $\lambda_{j}$ можно выбрать таким образом, чтобы $\mu_{1}, \mu_{2}$ не принадлежали $\Gamma(X) * I(X)$. Требуемое утверждение в случае А доказано.

В. Либо $|p|=1$, либо $|q|=1$. Пусть для определенности $|p|=1$. Не ограничивая общности, можно считать, что $p=1$. Пусть $q=q_{1} q_{2}$, где $\left|q_{j}\right|>1$. Очевидно, что если $f_{q} \in \operatorname{Aut}(X)$, то и $f_{q_{1}}, f_{q_{2}} \in \operatorname{Aut}(X)$. Поэтому независимость линейных статистик $L_{1}=\xi_{1}+\xi_{2}$ и $L_{2}=\xi_{1}+q \xi_{2}$ равносильна независимости $L_{1}=\xi_{1}+\xi_{2}$ и $L_{2}=\left(q_{1}\right)^{-1} \xi_{1}+q_{2} \xi_{2}$. Делая замену $\xi_{1}^{\prime}=\left(q_{1}\right)^{-1} \xi_{1}$, мы сводим задачу к случаю, когда $L_{1}=q_{1} \xi_{1}+$ $\xi_{2}, L_{2}=\xi_{1}+q_{2} \xi_{2}$. Соответствующее функциональное уравнение для характеристических функций $\widehat{\mu}_{j}(y)$ в этом случае имеет вид

$$
\widehat{\mu}_{1}\left(q_{1} u+v\right) \widehat{\mu}_{2}\left(u+q_{2} v\right)=\widehat{\mu}_{1}\left(q_{1} u\right) \widehat{\mu}_{2}(u) \widehat{\mu}_{1}(v) \widehat{\mu}_{2}\left(q_{2} v\right), \quad u, v \in Y .
$$

Пусть $\lambda_{j} \in M^{1}(G)$ и $\sigma\left(\lambda_{j}\right) \subset A\left(G, H^{\left(q_{j}\right)}\right), j=1,2$. Тогда $\hat{\lambda}_{j}\left(u+q_{j} v\right)=$ $\widehat{\lambda}_{j}(u)$ при $u, v \in H$ и характеристические функции $\widehat{\lambda}_{j}(y)$ удовлетворяют 
уравнению (5) при $u, v \in H$. Так же, как и в случае А, определяем функции $g_{j}(y)$ по формулам (3) и распределения $\mu_{j} \in M^{1}(X)$.

Проверим, что характеристические функции $\widehat{\mu}_{j}(y)$ удовлетворяют уравнению (5), а значит, линейные статистики $L_{1}$ и $L_{2}$ независимы. Рассуждаем так же, как в случае А. Если $u, v \in H$, то (5) выполнено. Пусть $u \notin H$. Тогда правая часть в (5) равна нулю. Если при этом левая часть в (5) отлична от нуля, то

$$
q_{1} u+v \in H \quad \text { и } \quad u+q_{2} v \in H .
$$

Отсюда получаем $s u \in H$, а значит, $u \in H$, что противоречит выбору $u$. Поэтому и левая часть в $(5)$ равна нулю. Случай $v \notin H$ рассматривается аналогично. Поскольку $\lambda_{j}$ можно выбрать таким образом, чтобы $\mu_{1}, \mu_{2}$ не принадлежали $\Gamma(X) * I(X)$, то требуемое утверждение в случае В доказано. Тем самым, утверждение теоремы 1 в случае 1 полностью доказано.

Случай 2: $|p q|=1$. Пусть для определенности $p=1$. Тогда $q=-1$ и линейные статистики $L_{1}$ и $L_{2}$ имеют вид $L_{1}=\xi_{1}+\xi_{2}, L_{2}=\xi_{1}-\xi_{2}$. Как доказано в [20], если $f_{2} \in \operatorname{Aut}(X)$, то из независимости $L_{1}$ и $L_{2}$ следует, что $\mu_{1}, \mu_{2} \in \Gamma(X) * I(X)$. Если же $f_{2} \notin \operatorname{Aut}(X)$, то существуют независимые случайные величины $\xi_{1}, \xi_{2}$ со значениями в $X$ и с распределениями $\mu_{1}, \mu_{2}$ такие, что $L_{1}$ и $L_{2}$ независимы, а $\mu_{1}, \mu_{2} \notin \Gamma(X) * I(X)$.

Случай 3: $p q-$ простое. Пусть для определенности $p=1$ и $q-$ простое, т.е. $L_{1}=\xi_{1}+\xi_{2}, L_{2}=\xi_{1}+q \xi_{2}$. Функциональное уравнение (2) для характеристических функций $\widehat{\mu}_{j}(y)$ принимает вид

$$
\widehat{\mu}_{1}(u+v) \widehat{\mu}_{2}(u+q v)=\widehat{\mu}_{1}(u) \widehat{\mu}_{2}(u) \widehat{\mu}_{1}(v) \widehat{\mu}_{2}(q v), \quad u, v \in Y .
$$

Имеются следующие возможности.

А. Либо $f_{s_{j}} \notin \operatorname{Aut}(X)$ хотя бы при одном $s_{j} \neq 2$, либо $f_{2} \notin \operatorname{Aut}(X)$ и $s$ делится на 4 . Докажем, что тогда существуют независимые случайные величины $\xi_{1}, \xi_{2}$ со значениями в $X$ и с распределениями $\mu_{1}, \mu_{2}$ такие, что $L_{1}$ и $L_{2}$ независимы, а $\mu_{1}, \mu_{2} \notin \Gamma(X) * I(X)$. Пусть $f_{s_{j}} \notin \operatorname{Aut}(X)$ при некотором $s_{j} \neq 2$. Тогда $Y^{\left(s_{j}\right)} \neq Y$. Возьмем $y_{0} \notin Y^{\left(s_{j}\right)}$ и рассмотрим на группе $X$ плотность $\rho(x)=1+\operatorname{Re}\left(x, y_{0}\right) \geqslant 0$. Пусть $\mu_{1}, \mu_{2} \in M^{1}(X)-$ распределения с плотностью $\rho(x)$ относительно $m_{X}$. Тогда

$$
\widehat{\mu}_{j}(y)= \begin{cases}1, & y=0, \\ 2^{-1}, & y= \pm y_{0}, \\ 0, & y \notin\left\{0, \pm y_{0}\right\} .\end{cases}
$$

Проверим, что характеристические функции $\widehat{\mu}_{j}(y)$ удовлетворяют уравнению (6). Очевидно, что достаточно проверить справедливость (6) при 
$u \neq 0, v \neq 0$. Тогда правая часть в (6) равна нулю. Если левая часть в (6) отлична от нуля, то

$$
u+v \in\left\{0, \pm y_{0}\right\} \quad \text { и } \quad u+q v \in\left\{0, \pm y_{0}\right\} .
$$

Отсюда

$$
s v \in\left\{0, \pm y_{0} \pm 2 y_{0}\right\} .
$$

Поскольку 2 и $s_{j}$ взаимно просты и $y_{0} \notin Y^{\left(s_{j}\right)}$, то $2 y_{0} \notin Y^{\left(s_{j}\right)}$, а следовательно, выполнение (7) невозможно. Значит, левая часть в (6) также равна нулю.

Если $f_{2} \notin \operatorname{Aut}(X)$, то рассуждение совершенно аналогично. Нужно взять $y_{0} \notin Y^{(2)}$, а невозможность выполнения (7) следует из того, что $s$ делится на 4.

Итак, уравнение (6) выполнено при всех $u, v \in Y$. Следовательно, линейные статистики $L_{1}$ и $L_{2}$ независимы. По построению $\mu_{1}, \mu_{2} \notin$ $\Gamma(X) * I(X)$. Тем самым, требуемое утверждение в случае А доказано.

B. $f_{s} \in \operatorname{Aut}(X)$. Докажем, что в этом случае по крайней мере одно из распределений $\mu_{j}$ принадлежит $\Gamma(X) * I(X)$. Для доказательства нам понадобятся несколько лемм.

Лемма 1. Пусть $Y-$ произвольная абелева группа, $q \in \mathbf{Z}, q \neq 0$,

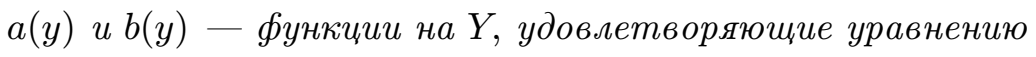

$$
a(u+v) b(u+q v)=a(u) b(u) a(v) b(q v), \quad u, v \in Y,
$$

и условиям

$$
a(-y)=a(y), \quad b(-y)=b(y), \quad y \in Y .
$$

Положим $s=1-q$. Если при некотором $y_{0} \in Y^{(s)}$ выполнено $a\left(y_{0}\right) b\left(y_{0}\right) \neq 0$, то найдется такая подгруппа $M \subset Y$, ито $a(y) b(y) \neq 0$ при $y \in M$.

Д о к а з а т е ль с т в о. Полагая в (8) сначала $u=-q y, v=y$, затем $u=y, v=-y$ и учитывая (9), получаем

$$
\begin{gathered}
a(s y)=a(q y) b^{2}(q y) a(y), \quad y \in Y, \\
b(s y)=a^{2}(y) b(y) b(q y), \quad y \in Y .
\end{gathered}
$$

По условию $y_{0}=s z_{0}$. Из (10) и (11) находим

$$
a\left(z_{0}\right) \neq 0, \quad a\left(q z_{0}\right) \neq 0, \quad b\left(z_{0}\right) \neq 0, \quad b\left(q z_{0}\right) \neq 0 .
$$

Из (8), полагая вначале $u=z_{0}, v=k z_{0}$, а затем $u=q z_{0}, v=k z_{0}, k \in \mathbf{Z}$, получаем

$$
\begin{aligned}
& a\left((k+1) z_{0}\right) b\left((k q+1) z_{0}\right)=a\left(z_{0}\right) b\left(z_{0}\right) a\left(k z_{0}\right) b\left(k q z_{0}\right), \\
& a\left((q+k) z_{0}\right) b\left((k+1) q z_{0}\right)=a\left(q z_{0}\right) b\left(q z_{0}\right) a\left(k z_{0}\right) b\left(k q z_{0}\right) .
\end{aligned}
$$


Учитывая (12), из (13) и (14) по индукции получаем, что $a\left(k z_{0}\right) \neq 0$, $b\left(k q z_{0}\right) \neq 0, k \in \mathbf{Z}$. Подгруппа $M=\left\{k q z_{0}\right\}_{k \in \mathbf{Z}}$ является искомой. Лемма 1 доказана.

Лемма 2. Пусть $M$ - произвольная абелева группа, функиии а $(y)$ u b(y) определены на $M$, удовлетворяют уравнению (8), условиям (9) и

$$
0<a(y) \leqslant 1, \quad 0<b(y) \leqslant 1, \quad a(0)=b(0)=1 .
$$

Положим $s=1-q$. Тогда на подгруппе $M^{(s q)}$ имеют место представления

$$
a(y)=\exp \left\{-\varphi_{1}(y)\right\}, \quad b(y)=\exp \left\{-\varphi_{2}(y)\right\},
$$

где $\varphi_{j}(y) \geqslant 0$ и $\varphi_{j}(y)$ удовлетворяют уравнению (1).

Следствие 1. Пусть в условиях леммь $2 M-$ подгруппа в $\mathbf{Q}$. Тогда имеют место представления

$$
a(y)=e^{-\sigma_{1} y^{2}}, \quad b(y)=e^{-\sigma_{2} y^{2}}, \quad \sigma_{1} \geqslant 0, \quad \sigma_{2} \geqslant 0, \quad y \in M^{(s q)} .
$$

Доказ а тельст в о ле м мы 2. Положим $\varphi_{1}(y)=-\ln a(y)$, $\varphi_{2}(y)=-\ln b(y)$. Из (8) получаем

$$
\varphi_{1}(u+v)+\varphi_{2}(u+q v)=A(u)+B(v), \quad u, v \in M,
$$

где $A(u)=\varphi_{1}(u)+\varphi_{2}(u), B(v)=\varphi_{1}(v)+\varphi_{2}(q v)$. Применим для решения уравнения (17) метод конечных разностей. Пусть $h \in M$. Для произвольной функции $g(y)$ на $M$ положим

$$
\Delta_{h} g(y)=g(y+h)-g(y) .
$$

Придадим $u$ и $v$ в (17) приращения $h_{1}$ и $k_{1}$ соответственно, причем так, чтобы выполнялось равенство $h_{1}+q k_{1}=0$. Вычитая уравнение (17) из полученного уравнения, находим

$$
\varphi_{1}\left(u+v+l_{1}\right)-\varphi_{1}(u+v)=\Delta_{h_{1}} A(u)+\Delta_{k_{1}} B(v),
$$

где $l_{1}=s k_{1}$. Полагая в (18) $v=0$ и вычитая из (18) полученное равенство, имеем

$$
\varphi_{1}\left(u+v+l_{1}\right)-\varphi_{1}(u+v)-\varphi_{1}\left(u+l_{1}\right)+\varphi_{1}(u)=\Delta_{k_{1}} B(v)-\Delta_{k_{1}} B(0) .
$$

Положим здесь $v=l_{1}$ и отметим, что $l_{1}-$ произвольный элемент подгруппы $M^{(s)}$. Из (19) получаем

$$
\Delta_{l_{1}}^{2} \varphi_{1}(u)=\varepsilon_{1}\left(l_{1}\right), \quad u \in M, \quad l_{1} \in M^{(s)},
$$

где $\varepsilon_{1}(y)$ - некоторая функция на $M^{(s)}$. Из $(20)$ находим

$$
\Delta_{l_{1}}^{3} \varphi_{1}(u)=0, \quad u, l_{1} \in M^{(s)} .
$$


Учитывая (9) и $(15)$, из $(21)$ легко получаем, что $\varphi_{1}(y) \geqslant 0$ и $\varphi_{1}(y)$ удовлетворяет уравнению (1).

Рассуждая аналогично, мы приходим к следующему уравнению для функции $\varphi_{2}(y)$ :

$$
\varphi_{2}\left(u+q v+l_{2}\right)-\varphi_{2}(u+q v)-\varphi_{2}\left(u+l_{2}\right)+\varphi_{2}(u)=\Delta_{k_{2}} B(v)-\Delta_{k_{2}} B(0),
$$

где $l_{2}=-s k_{2}$. Рассмотрим уравнение $(22)$, предполагая, что $k_{2} \in M^{(q)}$. Тогда $l_{2}$ - произвольный элемент подгруппы $M^{(s q)}$ и можно положить $q v=l_{2}$. В результате получаем

$$
\Delta_{l_{2}}^{2} \varphi_{2}(u)=\varepsilon_{2}\left(l_{2}\right), \quad u \in M, \quad l_{2} \in M^{(s q)},
$$

где $\varepsilon_{2}\left(l_{2}\right)$ - некоторая функция на $M^{(s q)}$. Из $(23)$ получаем

$$
\Delta_{l_{2}}^{3} \varphi_{2}(u)=0, \quad u, l_{2} \in M^{(s q)} .
$$

Учитывая (9) и $(15)$, из $(24)$ легко получаем, что $\varphi_{2}(y) \geqslant 0$ и $\varphi_{2}(y)$ удовлетворяет уравнению (1). Лемма 2 доказана.

Перейдем теперь к рассмотрению случая В. Очевидно, что характеристические функции распределений $\bar{\mu}_{j}$ также удовлетворяют уравнению (6). Поэтому уравнению (6) удовлетворяют и характеристические функции распределений $\nu_{j}=\mu_{j} * \bar{\mu}_{j}$. При этом $\widehat{\nu}_{j}(y)=\left|\widehat{\mu}_{j}(y)\right|^{2} \geqslant 0$, $j=1,2$. Положим $a(y)=\widehat{\nu}_{1}(y), b(y)=\widehat{\nu}_{2}(y)$. Очевидно, что функции $a(y)$ и $b(y)$ удовлетворяют уравнению (8) и условиям (9).

Предположим вначале, что при любом $y \in Y, y \neq 0$ выполнено $a(y) b(y)=0$. Из (10) тогда следует, что $a(s y)=0$ при любом $y \in Y$, $y \neq 0$. Поскольку $f_{s} \in \operatorname{Aut}(X)$, то $a(y)=0$ при любом $y \in Y, y \neq 0$. Следовательно, $\nu_{1}=m_{X}$, а значит и $\mu_{1}=m_{X}$. Аналогично из (11) получаем, что $\mu_{2}=m_{X}$.

Предположим теперь, что при некотором $y_{0} \in Y, y_{0} \neq 0$ выполнено $a\left(y_{0}\right) b\left(y_{0}\right) \neq 0$. Рассмотрим отдельно два случая: $q>0$ и $q<0$.

(i): $q>0$. Поскольку $Y^{(s)}=Y$, мы можем применить лемму 1 и получить подгруппу $M \subset Y$, на которой $a(y) b(y) \neq 0$. Сужения характеристических функций $a(y)$ и $b(y)$ на $M$ удовлетворяют условиям следствия 1. Поэтому на $M^{(s q)}$ мы имеем представление (16). Подставляя (16) в (8), получаем $\sigma_{1}+q \sigma_{2}=0$. Следовательно, $\sigma_{1}=\sigma_{2}=0$. Таким образом, на подгруппе $M^{(s q)}$ выполнено: $a(y)=b(y)=1$. Обозначим $E=\{y \in Y: a(y)=b(y)=1\}$. Тогда $E-$ ненулевая подгруппа в $Y$ и при любых $y \in Y, h \in E$ справедливы равенства

$$
a(y+h)=a(y), \quad b(y+h)=b(y) .
$$

Поэтому мы можем перейти от уравнения (8) на $Y$ к индуцированному уравнению на фактор-группе $L=Y / E$. Сохраним обозначения $a(y)$ и 
$b(y)$ для индуцированных функций. Отметим, что

$$
\{y \in L: a(y)=b(y)=1\}=\{0\} .
$$

Из (11) следует, что $f_{s}-$ мономорфизм на $L$. Поскольку $L-$ периодическая группа, то $f_{s} \in \operatorname{Aut}(L)$.

Из (10) вытекает, что

$$
a(s y) \leqslant a(y), \quad y \in L .
$$

Так как любой элемент группы $L$ имеет конечный порядок, то для любого $y \in L$ выполнено $s^{n} y=y$ при некотором натуральном $n$, зависящем, вообще говоря, от $y$. Из (26) тогда следует, что

$$
a(y)=a\left(s^{n} y\right) \leqslant \cdots \leqslant a(s y) \leqslant a(y), \quad y \in L .
$$

Таким образом, на каждой орбите $\left\{y, s y, \ldots, s^{n-1} y\right\}$ функция $a(y)$ принимает постоянное значение. Из (11) вытекает аналогичное утверждение для $b(y)$.

Предположим, что в некоторой точке $y_{0} \in L, y_{0} \neq 0$ выполнено $a\left(y_{0}\right) \neq 0$. Тогда $a\left(s y_{0}\right)=a\left(y_{0}\right) \neq 0$ и из $(10)$ получаем

$$
a\left(q y_{0}\right)=b\left(q y_{0}\right)=1 \text {. }
$$

Из $(25)$ и $(27)$ следует, что $q y_{0}=0$. Аналогично, из (11) находим, что если $b\left(y_{0}\right) \neq 0, y_{0} \in L, y_{0} \neq 0$, то $a\left(y_{0}\right)=b\left(q y_{0}\right)=1$, и значит, (27) также выполнено. Заметим теперь, что любая фактор-группа группы $Y$, в частности $L$, содержит не более одной подгруппы $A \approx \mathbf{Z}(q)$. Пусть $A$ - подгруппа в $L$, порожденная элементом $y_{0}$. Рассмотрим сужение уравнения (8) на $A$. Учитывая, что $q y=0$ для любого $y \in A$, получаем

$$
a(u+v) b(u)=a(u) b(u) a(v), \quad u, v \in A .
$$

Если в некоторой точке $u_{0} \in A, u_{0} \neq 0$ выполнено $b\left(u_{0}\right) \neq 0$, то из (28) следует, что

$$
a\left(u_{0}+v\right)=a\left(u_{0}\right) a(v), \quad v \in A .
$$

Полагая здесь $v=(q-1) u_{0}$, получаем $a\left(u_{0}\right)=1$, а поскольку $q-$ простое число, то $a(y) \equiv 1, y \in A$. Если же $b(y)=0$ при любом $y \in A, y \neq 0$, то, очевидно, функция $a(y)$ может быть произвольной. Тем самым, доказано, что либо

$$
a(y)= \begin{cases}1, & y \in A, \\ 0, & y \notin A,\end{cases}
$$

либо

$$
b(y)= \begin{cases}1, & y=0 \\ 0, & y \neq 0\end{cases}
$$


Возвращаясь от индуцированных функций на $L$ к функциям $a(y)$ и $b(y)$ на $Y$, получаем, что либо $\nu_{1} \in I(X)$, либо $\nu_{2} \in I(X)$, а значит, либо $\mu_{1} \in I(X)$, либо $\mu_{2} \in I(X)$.

Если же в любой точке $y_{0} \in L, y_{0} \neq 0$ выполнено $a\left(y_{0}\right)=b\left(y_{0}\right)=0$, то справедливо представление (29) для функции $a(y)$, где $A=\{0\}$, и представление (30) для функции $b(y)$. В этом случае, очевидно, $\mu_{1}, \mu_{2} \in$ $I(X)$. Случай $q>0$, таким образом, полностью исследован.

(ii): $q<0$. Нам понадобятся для дальнейшего следующие леммы.

Лемма 3 ([21]). Пусть $X-$ локально компактная сепарабельная абелева метрическая группа, не содержащая подгруппь, топологически изоморфной $\mathbf{T}, \mu \in \Gamma(X), \mu=\mu_{1} * \mu_{2}$, где $\mu_{j} \in M^{1}(X)$. Тогда $\mu_{j} \in \Gamma(X), j=1,2$.

Лемма 4. Пусть $X=\Sigma_{\mathbf{a}}, \mu \in M^{1}(X) u \nu=\mu_{1} * \bar{\mu}$. Тогда, если $\nu=\gamma * m_{K}$, где $\gamma \in \Gamma(X)$, a $A(Y, K) \not \approx \mathbf{Z}$, mo $\mu \in \Gamma(X) * I(X)$.

Д о к а з а т е л ь с т в о. Обозначим $L=A(Y, K)$. Сужение характеристической функции $\widehat{\nu}(y)$ на $L$ является характеристической функцией некоторого гауссовского распределения на фактор-группе $X / K$. Поскольку $L \not \approx \mathbf{Z}$, то $X / K \not \approx \mathbf{T}$. Очевидно также, что фактор-группа $X / K$ не может содержать подгруппы, топологически изоморфной $\mathbf{T}$. Из леммы 3 тогда следует, что сужение характеристической функции $\widehat{\mu}(y)$ на $L$ является характеристической функцией некоторого гауссовского распределения. Следовательно, мы имеем представление

$$
\widehat{\mu}(y)= \begin{cases}\left(\left[x_{0}\right], y\right) \exp \{-\varphi(y)\}, & y \in L, \\ 0, & y \notin L,\end{cases}
$$

где $\left[x_{0}\right] \in X / K$, а $\varphi(y)=\sigma y^{2}$. Функция $\varphi(y)$ естественным образом продолжается с $L$ на $Y$. Возьмем $x_{0} \in\left[x_{0}\right]$ и рассмотрим распределение $\lambda \in \Gamma(X)$ с характеристической функцией

$$
\widehat{\lambda}(y)=\left(x_{0}, y\right) \exp \{-\varphi(y)\}, \quad y \in Y .
$$

Из (31) следует, что $\mu=\lambda * m_{K}$. Лемма 4 доказана.

Лемма 5. Пусть $Y-$ nодгруппа в $\mathbf{Q}, F-$ nодгруппа в $Y$. Pac-

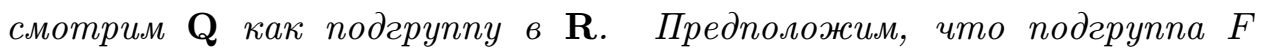
плотна в $\mathbf{R}$ в топологии $\mathbf{R}$, т.е. в топологии, индуцированной на $F$ обычной топологией $\mathbf{R}$. Пусть $g(y)$ - положительно определенная функция на $Y$ такая, что ее сужение на $F$ - непрерьвная функция $в$ топологии R. Тогда:

(a) для любого $u \in Y$ сужение функции $g(y)$ на класс смежности $u+F$ является равномерно непрерьвной функиией в топологии $\mathbf{R}$;

(b) для любого $u \in Y$ существует предел

$$
g_{u}=\lim _{y \rightarrow 0, y \in u+F} g(y)
$$


(c) если при некотором $u \in Y$ выполнено $g_{u}=1$, то функиия $g(y)$ будет равномерно непрерьвной в топологии $\mathbf{R}$ на подгруппе $V$, порожденной и и $F$.

Д о к а з а т е л ь с т в о. Утверждение (a) следует из неравенства

$$
|h(u)-h(v)|^{2} \leqslant 2(1-\operatorname{Re} h(u-v)),
$$

справедливого для любой положительно определенной функции $h(y)$. Утверждение (b) непосредственно вытекает из (a). Для доказательства (c) воспользуемся неравенством

$$
1-\operatorname{Re} h(u+v) \leqslant 2[(1-\operatorname{Re} h(u))+(1-\operatorname{Re} h(v))],
$$

также справедливым для произвольной положительно определенной функции $h(y)$. Из (33) следует, что если $g_{u}=1$, то $g_{v}=1$ для любого $v \in V$. Поскольку фактор-группа $V / F$ конечна, то равномерная непрерывность сужения функции $g(y)$ на $V$ вытекает из (32). Лемма 5 доказана.

Перейдем теперь к рассмотрению случая $q<0$. Из (11) вытекает, что при любом натуральном $m$ выполнено

$$
a\left(\frac{y_{0}}{s^{m}}\right) b\left(\frac{y_{0}}{s^{m}}\right) \neq 0
$$

Из доказательства леммы 1 следует, что $a(y) b(y) \neq 0$ на подгруппе $y_{0} H^{(q)}$, а тогда по следствию 1 на подгруппе $y_{0} H^{\left(q^{2}\right)}$ имеет место представление (16). Пусть $F$ - максимальная подгруппа в $Y$, содержащая $y_{0} H^{\left(q^{2}\right)}$, на которой имеет место представление (16). Поскольку $F \not \approx \mathbf{Z}$, то $F$ плотна в $\mathbf{R}$ в топологии $\mathbf{R}$. Из (16) по лемме 5 , примененной к функциям $a(y)$ и $b(y)$, следует, что для любого $u \in Y$ существуют пределы

$$
a_{u}=\lim _{y \rightarrow 0, y \in u+F} a(y), \quad b_{u}=\lim _{y \rightarrow 0, y \in u+F} b(y) .
$$

Заметим теперь, что полученные функции $a_{u}$ и $b_{u}$ по построению принимают постоянные значения на каждом классе смежности $u+F$ и определяют поэтому некоторые функции $a_{[u]}$ и $b_{[u]}$ на фактор-группе $L=Y / F$. Функции $a_{[u]}$ и $b_{[u]}$, очевидно, удовлетворяют уравнению (8) и условиям (9), а значит, и уравнениям (10) и (11). Поскольку $F$ - максимальная подгруппа в $Y$, на которой имеет место представление (16), то в силу утверждения (c) леммы 5

$$
\left\{[u] \in L: a_{[u]}=b_{[u]}=1\right\}=\{0\},
$$

что является аналогом (25). Поскольку $L-$ периодическая группа, то из (11) и леммы 3 вытекает, что $f_{s} \in \operatorname{Aut}(L)$. Повторяя теперь доказательство для случая (i), мы получаем либо представление (29) для функции $a_{[u]}$, либо представление $(30)$ для функции $b_{[u]}$. 
Заметим теперь, что если $a_{[u]}=0$ при любом $[u] \notin A$, то $a(y)=0$ при любом $y \notin f_{q}^{-1}(F)$. Действительно, подставим в $(8) u \in F, v_{0} \notin f_{q}^{-1}(F)$. Учитывая (16), получаем

$$
a\left(u+v_{0}\right) b\left(u+q v_{0}\right)=e^{-\sigma_{1} u^{2}-\sigma_{2} q^{2} u^{2}} a\left(v_{0}\right) b\left(q v_{0}\right) .
$$

Перейдем здесь к пределу при $u \rightarrow-v_{0}, u \in F$. Поскольку $\left[v_{0}\right] \notin A$, мы имеем

$$
0=e^{-\sigma_{1} v_{0}^{2}-\sigma_{2} q^{2} v_{0}^{2}} a\left(v_{0}\right) b\left(q v_{0}\right),
$$

а значит, $a\left(v_{0}\right) b\left(q v_{0}\right)=0$. Из (10) тогда следует, что $a\left(s v_{0}\right)=0$. Заметим теперь, что $f_{s} \in \operatorname{Aut}(F)$. Действительно, из (11) по лемме 3 следует, что на подгруппе $f_{s}^{-1}(F)$ справедливо представление (16). Значит, $f_{s}^{-1}(F)=F$, поскольку $F$ - максимальная подгруппа в $Y$, на которой справедливо (16). А тогда $f_{s}(F)=F$, что эквивалентно $f_{s} \in \operatorname{Aut}(F)$. Отсюда следует, что $f_{s}$ взаимно однозначно отображает множество $f_{q}^{-1}(F)$ в себя. Поэтому из равенства $a(s v)=0$ при любом $v \notin f_{q}^{-1}(F)$ следует, что $a(v)=0$ при любом $v \notin f_{q}^{-1}(F)$. В результате из представления (29) для функции $a_{[u]}$ получаем

$$
a(y)= \begin{cases}e^{-\sigma_{1} y^{2}}, & y \in f_{q}^{-1}(F), \\ 0, & y \notin f_{q}^{-1}(F) .\end{cases}
$$

Рассуждая аналогично, убеждаемся, что если $b_{[u]}=0$ при любом $[u] \neq 0$, то $b(y)=0$ при любом $y \notin F$. А тогда из представления (30) для функции $b_{[u]}$ следует представление

$$
b(y)= \begin{cases}e^{-\sigma_{2} y^{2}}, & y \in F, \\ 0, & y \notin F .\end{cases}
$$

Таким образом, по крайней мере одно из распределений $\nu_{j}$ принадлежит $\Gamma(X) * I(X)$, а тогда по лемме 4 и соответствующее распределение $\mu_{j}$ принадлежит $\Gamma(X) * I(X)$. Случай В в целом полностью исследован.

C. $f_{s_{j}} \in \operatorname{Aut}(X)$ при всех $s_{j} \neq 2, f_{2} \notin \operatorname{Aut}(X)$ и $s$ не делится на 4. Докажем, что этом случае по крайней мере одно из распределений $\mu_{j}$ принадлежит $\Gamma(X) * I(X)$. Поскольку $s$ делится на 2 и не делится на 4 , то $q=4 k-1, k \in \mathbf{Z}$. Пусть, как и в случае $\mathrm{B}, \nu_{j}=\mu_{j} * \bar{\mu}_{j}, j=1,2$, $a(y)=\widehat{\nu}_{1}(y), b(y)=\widehat{\nu}_{2}(y)$. Имеются две возможности.

(i). Предположим, что выполнено

$$
a(y) b(y)=0, \quad y \in Y^{(2)}, \quad y \neq 0 .
$$

Полагая в (8) сначала $u=q y, v=y$, затем $u=v=y$ и учитывая (10) и (11), получаем

$$
\begin{aligned}
& a(s y)=a((q+1) y) b(2 q y), \quad y \in Y, \\
& b(s y)=a(2 y) b((q+1) y), \quad y \in Y .
\end{aligned}
$$


Поскольку $s=2-4 k$, то из (37) и (38) следует

$$
\begin{gathered}
a((1-2 k) y)=a(2 k y) b(q y), \quad y \in Y, \\
b((1-2 k) y)=a(y) b(2 k y), \quad y \in Y .
\end{gathered}
$$

Отсюда

$$
a((1-2 k) y) b((1-2 k) y)=a(2 k y) b(2 k y) a(y) b(q y), \quad y \in Y .
$$

Поскольку $1-2 k-$ делитель $s$, то $f_{1-2 k} \in \operatorname{Aut}(X)$ и из (41) и (36) следует, что $a(y) b(y)=0$ при любом $y \in Y, y \neq 0$. Но тогда из (10) и (11) вытекает, что $a(y)=b(y)=0$ при любом $y \in Y^{(2)}, y \neq 0$. Из (39) и (40) следует, что $\nu_{1}=\nu_{2}=m_{X}$, а значит, $\mu_{1}=\mu_{2}=m_{X}$.

(ii). Предположим, что (36) не выполнено, т.е. $a\left(y_{0}\right) b\left(y_{0}\right) \neq 0$ при некотором $y_{0} \in Y^{(2)}, y_{0} \neq 0$. При $q>0$ без изменений проходит доказательство для случая $\mathrm{B}(\mathrm{i})$, так как из равенства $Y^{(s)}=Y^{(2)}$ следует, что функции $a(y)$ и $b(y)$ удовлетворяют условиям леммы 1.

При $q<0$ доказательство проводится по схеме доказательства для случая $\mathrm{B}(\mathrm{ii})$, но требует некоторых пояснений, поскольку $f_{s} \notin \operatorname{Aut}(X)$. Мы сохраним обозначения, использованные при доказательстве случая $\mathrm{B}(\mathrm{ii})$.

Для доказательства того, что существует подгруппа $W \not \approx \mathbf{Z}$, на которой справедливо представление (16), рассуждаем следующим образом. Пусть на подгруппе $M$, порожденной некоторым элементом $\zeta$, выполнено $a(y) b(y) \neq 0$ (существование такой подгруппы гарантировано леммой 1$)$. Подставим в $(8)$

$$
u=\frac{l}{1-2 k} \zeta, \quad v=\left(1-\frac{l}{1-2 k}\right) \zeta, \quad l \in \mathbf{Z} .
$$

При этих $u$ и $v$ выполнено $u+v, u+q v \in M$. Следовательно, левая часть в (8) отлична от нуля. Значит, $a\left(l(1-2 k)^{-1} \zeta\right) b\left(l(1-2 k)^{-1} \zeta\right) \neq 0$. Поскольку $f_{1-2 k} \in \operatorname{Aut}(X)$, то мы получаем, что на подгруппе $S=$ $\zeta H$ выполнено $a(y) b(y) \neq 0$. Так как $q \neq-1$, то $H \not z \mathbf{Z}$, а значит, и $S \not \approx \mathbf{Z}$. Далее доказательство проводится без изменений так же, как и в случае В(ii), и мы получаем либо представление (29) для функции $a_{[u]}$, либо представление (30) для функции $b_{[u]}$.

Чтобы получить искомые представления, (34) для функции $a(y)$ или $(35)$ для функции $b(y)$, достаточно убедиться, что если $a(s y)=0$ при всех $y \notin f_{q}^{-1}(F)$, то $a(y)=0, y \notin f_{q}^{-1}(F)$, а также что если $b(s y)=0$ при всех $y \notin F$, то $b(y)=0, y \notin F$.

Мы ограничимся рассуждением для функции $a(y)$. Для функции $b(y)$ доказательство аналогично. Пусть $y_{0} \notin f_{q}^{-1}(F)$. Рассмотрим соответствующий класс смежности $y_{0}+F$ и отметим, что $q\left[y_{0}\right] \neq 0$. Поскольку $f_{s} \in \operatorname{Aut}(L)$, то

$$
s\left[y^{\prime}\right]=\left[y_{0}\right]
$$


при некотором $\left[y^{\prime}\right] \in L$. Легко видеть, что $y^{\prime} \notin f_{q}^{-1}(F)$, так как в противном случае было бы выполнено равенство $q\left[y^{\prime}\right]=0$ и, учитывая (42), мы имели бы равенство $\left[y^{\prime}\right]=\left[y_{0}\right]$, что противоречит выбору $y_{0}$. Из (42) следует, что $s y^{\prime}=y_{0}+h, h \in F$. Но тогда при любом $h^{\prime} \in F$ выполнено $s\left(y^{\prime}+h^{\prime}\right) \in y_{0}+F$. Поскольку $F \not z \mathbf{Z}$, то множество $s\left(y^{\prime}+F\right)$ плотно в $y_{0}+F$ в топологии $\mathbf{R}$. Функция $a(y)$ равномерно непрерывна на $y_{0}+F$ в топологии $\mathbf{R}$ и по условию $a\left(s\left(y^{\prime}+h^{\prime}\right)\right)=0$ при любом $h^{\prime} \in F$. Значит, $a(y) \equiv 0, y \in y_{0}+F$, в частности, $a\left(y_{0}\right)=0$. Требуемое утверждение в случае (ii) доказано, а значит, доказано и утверждение теоремы 1 в случае 3. Тем самым, теорема 1 полностью доказана.

3 а м е ч а н и е 1. Рассуждение, проведенное при рассмотрении случая 3 , показывает, что в случаях $3 \mathrm{~B}$ и $3 \mathrm{C}$ у уравнения (6) всегда существуют такие решения $\widehat{\mu}_{j}(y)$, где одно из распределений $\mu_{j}$ не принадлежит $\Gamma(X) * I(X)$. Следовательно, утверждение теоремы 1 в случаях $3 \mathrm{~B}$ и $3 \mathrm{C}$ не может быть усилено.

3 а м е ч а н и е 2 . Пусть $X-$ связная локально компактная сепарабельная абелева метрическая группа, $\xi_{1}, \xi_{2}$ - независимые случайные величины со значениями в $X$ и с распределениями $\mu_{1}, \mu_{2}$. Как доказано в [20], условие

$$
f_{2} \in \operatorname{Aut}(X)
$$

является необходимым и достаточным для того, чтобы из независимости линейных статистик $L_{1}=\xi_{1}+\xi_{2}$ и $L_{2}=\xi_{1}-\xi_{2}$ следовало, что $\mu_{1}$, $\mu_{2} \in \Gamma(X) * I(X)$. Из теоремы 1 вытекает, что справедливы следующие утверждения:

$(\alpha)$ для а-адического соленоида $X=\Sigma_{\mathbf{a}}$ условие (i) является необходимым для того, чтобы существовали автоморфизмы $\alpha_{j}, \beta_{j} \in \operatorname{Aut}(X)$ такие, что из независимости линейных статистик $L_{1}=\alpha_{1} \xi_{1}+\alpha_{2} \xi_{2}$ и $L_{2}=\beta_{1} \xi_{1}+\beta_{2} \xi_{2}$ следует, что $\mu_{1}, \mu_{2} \in \Gamma(X) * I(X)$;

$(\beta)$ если для а-адического соленоида $X=\Sigma_{\mathbf{a}}$ условие (i) выполнено, то не существует линейных статистик, отличных от $L_{1}=\xi_{1}+\xi_{2}$ и $L_{2}=\xi_{1}-\xi_{2}$, независимость которых влечет, что $\mu_{1}, \mu_{2} \in \Gamma(X) * I(X)$.

Утверждения $(\alpha)$ и $(\beta)$, вообще говоря, неверны для произвольных связных компактных абелевых групп. Для построения соответствующего контрпримера к утверждению $(\alpha)$ положим $X=X_{1} \times X_{2}$, где $X_{j}=\Sigma_{\mathbf{a}_{j}}, \mathbf{a}_{1}=(2,2, \ldots), \mathbf{a}_{2}=(3,3, \ldots)$. Очевидно, что группа $X$ связна и $f_{2} \notin \operatorname{Aut}(X)$. Пусть $\alpha \in \operatorname{Aut}(X), \alpha\left(x_{1}, x_{2}\right)=\left(-x_{1}, x_{2}\right), x_{j} \in X_{j}$ и $\xi_{j}-$ независимые случайные величины со значениями в $X$ и с распределениями $\mu_{j}$. Проверим, что если $L_{1}=\xi_{1}+\xi_{2}$ и $L_{2}=\xi_{1}+\alpha \xi_{2}$ независимы, то $\mu_{1}, \mu_{2} \in \Gamma(X) * I(X)$.

Действительно, независимость $L_{1}$ и $L_{2}$ эквивалентна тому, что ха- 
рактеристические функции $\widehat{\mu}_{j}(y)$ удовлетворяют уравнению

$$
\widehat{\mu}_{1}(u+v) \widehat{\mu}_{2}(u+\widetilde{\alpha} v)=\widehat{\mu}_{1}(u) \widehat{\mu}_{2}(u) \widehat{\mu}_{1}(v) \widehat{\mu}_{2}(\widetilde{\alpha} v), \quad u, v \in Y,
$$

где $\widetilde{\alpha}\left(y_{1}, y_{2}\right)=\left(-y_{1}, y_{2}\right), y_{j} \in Y_{j}=X_{j}^{*}$. Положим $\nu_{j}=\mu_{j} * \bar{\mu}_{j}$. Тогда $\widehat{\nu}_{j}(y) \geqslant 0$ и характеристические функции $\widehat{\nu}_{j}(y)$ также удовлетворяют уравнению (43). Рассмотрим сужение уравнения (43) для функций $\widehat{\nu}_{j}(y)$ на подгруппу $Y_{2}$. Получаем

$$
\widehat{\nu}_{1}(u+v) \widehat{\nu}_{2}(u+v)=\widehat{\nu}_{1}(u) \widehat{\nu}_{2}(u) \widehat{\nu}_{1}(v) \widehat{\nu}_{2}(v), \quad u, v \in Y_{2} .
$$

Положим $g(y)=\widehat{\nu}_{1}(y) \widehat{\nu}_{2}(y)$. Из (44) тогда вытекает, что $g(y) \equiv 1, y \in Y_{2}$. Следовательно, и $\widehat{\nu}_{j}(y) \equiv 1, y \in Y_{2}, j=1,2$, а значит, $\sigma\left(\nu_{j}\right) \subset A\left(X, Y_{2}\right)=$ $X_{1}$. Распределения $\mu_{j}$ в таком случае можно заменить их сдвигами $\mu_{j}^{\prime}$ так, что $\sigma\left(\mu_{j}^{\prime}\right) \subset X_{1}$. Характеристические функции распределений $\mu_{j}^{\prime}$, очевидно, также удовлетворяют уравнению (43). Поскольку $\widehat{\mu}_{j}(y+h)=$ $\widehat{\mu}_{j}(y)$ для любых $y \in Y, h \in Y_{2}$, то достаточно решить уравнение (43), считая, что $u, v \in Y_{1}$. Но на $Y_{1}$ уравнение (43) принимает вид

$$
\widehat{\mu}_{1}^{\prime}(u+v) \widehat{\mu}_{2}^{\prime}(u-v)=\widehat{\mu}_{1}^{\prime}(u) \widehat{\mu}_{2}^{\prime}(u) \widehat{\mu}_{1}^{\prime}(v) \widehat{\mu}_{2}^{\prime}(-v), \quad u, v \in Y_{1} .
$$

Поскольку $f_{2} \in \operatorname{Aut}\left(X_{1}\right)$, то из (45) следует, что $\mu_{j}^{\prime} \in \Gamma\left(X_{1}\right) * I\left(X_{1}\right)$ (см. [20]), а значит, и $\mu_{j} \in \Gamma(X) * I(X)$.

Если в приведенном выше рассуждении в качестве $X$ взять $X=X_{1}^{2}$, то мы получим контрпример к утверждению $(\beta)$.

\section{СПИСОК ЛИТЕРАТУРЫ}

1. Скитович В. П. Об одном свойстве нормального распределения. - Докл. АН CCCP, 1953, т. 89, с. 217-219.

2. Darmois G. Analyse générale des liaisons stochastiques. - Rev. Inst. Internat. Statistique, 1953 , v. 21 , p. 2-8.

3. Ghurye S. G., Olkin I. A characterization of the multivariate normal distribution. Ann. Math. Statist., 1962, v. 33, p. 533-541.

4. Каган А. М., Линник Ю. В., Рао С. Р. Характеризационные задачи математической статистики. М.: Наука, 1972, 656 с.

5. Фельдман Г. М. Характеризация гауссовского распределения на группах независимостью линейных статистик. - Сиб. матем. журн., 1990, т. 31, № 2, с. 180-190.

6. Фельдман Г. М. К теореме Скитовича-Дармуа на абелевых группах. - Теория вероятн. и ее примен., 1992, т. 37, в. 4, с. 695-708.

7. Фельдман Г. М. Теорема Скитовича-Дармуа для компактных групп. - Теория вероятн. и ее примен., 1996, т. 41, в. 4, с. 901-906.

8. Фельдман Г. М. Теорема Скитовича-Дармуа для дискретных периодических абелевых групп. - Теория вероятн. и ее примен., 1997, т. 42, в. 4, с. 747-756.

9. Фельдман Г. М. К теореме Скитовича-Дармуа для конечных абелевых групп. Теория вероятн. и ее примен., 2000, т. 45, в. 3, с. 603-607.

10. Feldman G. M., Graczyk P. On the Skitovich-Darmois theorem on compact Abelian groups. - J. Theoret. Probab., 2000, v. 13, № 3, p. 859-869. 
11. Feldman G. M. A characterization of the Gaussian distribution on Abelian groups. Probab. Theory Related Fields, 2003, v. 126, № 1, p. 91-102.

12. Фельдман Г. М., Грачик П. К теореме Скитовича-Дармуа для дискретных абелевых групп. - Теория вероятн. и ее примен., 2004, т. 49, в. 3, с. 596-601.

13. Миронюк M.В., Фельдман Г. М. Независимые линейные статистики на двумерном торе. - Теория вероятн. и ее примен., 2007, т. 52, в. 1, с. 3-20.

14. Fel'dman G. M. Arithmetic of Probability Distributions, and Characterization Problems on Abelian Groups. Providence: Amer. Math. Soc., 1993, 223 p. (Transl. Math. Monogr., v. 116.)

15. Kagan A., Wesotowski J. An extension of the Darmois-Skitovitch theorem to a class of dependent random variables. - Statist. Probab. Lett., 2000, v. 47, № 1, p. 69-73.

16. Baryshnikov $Y$., Eisenberg B., Stadje $W$. Independent variables with independent sum and difference: $S^{1}$-case. - J. Multivariate Anal., 1993, v. 45, № 2, p. 161-170.

17. Parthasarathy K.R., Ranga Rao R., Varadhan S.R.S. Probability distributions on locally compact Abelian groups. - Illinois J. Math., 1963, v. 7, p. 337-369.

18. Хьюитm Э., Росс К. Абстрактный гармонический анализ, т. 1. М.: Наука, 1975, $656 \mathrm{c}$.

19. Хьюитm Э., Росс К. Абстрактный гармонический анализ, т. 2. М.: Мир, 1975, $901 \mathrm{c.}$

20. Фельдман Г. М. Гауссовские распределения в смысле Бернштейна на группах. Теория вероятн. и ее примен., 1986, т. 31, в. 1, с. 47-58.

21. Фельдман Г. М. О разложении гауссовского распределения. - Теория вероятн. и ее примен., 1977, т. 22, в. 1, с. 136-143.

Поступила в редакцию 